\title{
SR Research Square

\section{Putative positive role of inflammatory genes in fat deposition supported by altered gene expression in purified human adipocytes and preadipocytes from lean and obese adipose tissues}

\author{
Sang-Hyeop Lee \\ Kangwon National University \\ Nak-Hyeon Choi \\ Korea National Institute of Health \\ In-Uk Koh \\ Korea National Institute of Health \\ Bong-Jo Kim \\ Korea National Institute of Health
}

Song Lee

Asan Medical Center

Song-Cheol Kim

Asan Medical Center

Sun Shim Choi ( $\square$ schoi@kangwon.ac.kr)

Institute of bioscience and biotechnology https://orcid.org/0000-0001-6289-4220

Research article

Keywords: RNA-seq, adipocytes, preadipocytes, inflammatory genes, visceral adipose tissue

Posted Date: March 3rd, 2020

DOI: https://doi.org/10.21203/rs.3.rs-15748/v1

License: (c) (i) This work is licensed under a Creative Commons Attribution 4.0 International License. Read Full License 


\section{Abstract}

\section{Background}

Obesity is a chronic low-grade inflammatory disease that is generally characterized by enhanced inflammation in obese adipose tissue (AT). Here, we investigated alterations in gene expression between lean and obese conditions using mRNA-Seq data derived from human purified adipocytes (ACs) and preadipocytes (preACs).

Results

We defined four classes of differentially expressed genes (DEGs) by comparing gene expression between 1) lean and obese ACs, 2) lean and obese preACs, 3) lean ACs and lean preACs, and 4) obese ACs and obese preACs. Based on an analysis of comparison 1, numerous canonical obesity-related genes,

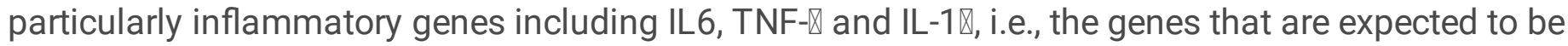
upregulated in obesity conditions, were found to be expressed at significantly lower levels in obese ACs than in lean ACs. In contrast, some inflammatory genes were found to be expressed at higher levels in obese preACs than lean preACs in the analysis of comparison 2. These two results indicate that (1) up-/downregulation of genes in ACs and preACs is inversely controlled during the fat deposition process and (2) preACs rather than ACs have increased inflammatory response genes in comparisons of lean and obese conditions for each of these cell types. Analysis of comparisons 3 and 4 showed that inflammatory gene classes were expressed at higher levels in differentiated ACs than undifferentiated preACs under both lean and obese conditions; however, the degree of upregulation was greater for lean than for obese conditions.

\section{Conclusions}

Taken together, our analyses may suggest that lean fat differentiation involves even greater enhancement of inflammatory responses than does obese fat differentiation.

\section{Background}

A widely accepted notion about obesity is that inflammatory responses are elevated in the serum as well as adipose tissue (AT) of obese organisms, as a so-called low-grade inflammatory disease [1]. AT is a primary organ that maintains homeostasis between energy uptake and energy expenditure, in which excess energy is stored in the form of triacylglycerols, whereas free fatty acids are released during fasting $[2,3]$. AT is also an endocrine organ that secretes various bioactive factors, namely, adipokines, that regulate the whole-body level of immune and inflammatory responses [4-6]. At the cellular level, obesity is defined as accelerated AT expansion and remodeling that induces either AT hypertrophy (i.e., adipocyte expansion due to excessive fat storage) or hyperplasia (i.e., increased adipogenesis from preadipocytes) through ECM remodeling and angiogenesis [7, 8]. Various ECM proteins, including MMP2, ADAM, TIMP, CTSK, and CTSS, are altered in obese AT [9-12]. Angiogenic genes such as VEGF and ANGPT2 are 
upregulated in response to activated HIF1A (i.e., hypoxia-related transcription factor) $[13,14]$. LEP and ADIPOQ are another genes that have potential adipokine functions involved in AT remodeling $[8,15]$.

Several studies have shown that the accumulation of excess fat in AT leads to the release of inflammatory mediators, such as TNF-a and IL-6, and the reduction of anti-inflammatory cytokines, such as adiponectin, is associated with chronic inflammation in obese individuals. It is also known that excess fat that is overflowed from AT can deposit in other organs such as liver, pancreas, and muscle, causing insulin resistance [16]. In addition, the oxidative stress due to excessive nutrients intake can contribute to increased inflammation associated with obesity [17]. The increased serum level of C-reactive protein (CRP) is one of the markers of chronic inflammation in obesity [18]. In fact, numerous studies strongly support the idea that the inflammatory responses mounted in AT and the accompanying extensive molecular and cellular changes are responsible for excess fat deposition associated with the metabolic pathogenicity of obesity such as diabetes and atherosclerosis $[19,20]$.

In contrast, a few recent studies provided an interesting view on the positive role of inflammatory responses in controlling adipogenesis. For instance, Ye and McGuinness [21] described that inflammatory responses are required for the maintenance of a healthy AT microenvironment for AT remodeling and expansion. By constructing three mouse models with adipocyte (AC)-specifically attenuated inflammatory responses, Asterholm et al. [22] showed that proper AT remodeling and expansion are executed by inflammatory responses at the level of ACs, so that reduced or impaired local inflammatory responses in the $A C$ cause pathogenic obesity-related conditions, such as hepatic steatosis or metabolic dysfunction due to ectopic lipid accumulation [23, 24]. Interestingly, a recent AT-derived RNA-seq analysis of pigs showed that pigs with thicker backfat tended to express significantly lower levels of immune and inflammatory genes than pigs with thinner back fat, which indicates that expression of high inflammatory genes may be associated with lower fat accumulation in AT [25]. Few studies have addressed the positive role of the inflammatory response which may have a role in fat deposition in lean individuals, although fat cell differentiation and fat deposition must be regulated appropriately to maintain healthy fat homeostasis.

We think that the contradictory conclusions regarding the positive or pathogenic role of inflammation in obesity are largely due to the use of differential experimental designs among different studies. As expected, retrieving lean (or healthy) AT samples is much more difficult than retrieving obese AT samples in humans. Purification of cells such as ACs, preadipocytes (preACs), macrophages, and endothelial cells residing in AT is even more difficult. For this reason, most transcriptome-based studies in humans have been unable to identify so-called differentially expressed genes (DEGs) by directly comparing lean and obese ATs from cohorts of lean and obese individuals. Instead, some studies have investigated DEGs in AT samples during weight loss induced by bariatric surgery for the same individuals [26], and others have analyzed DEGs between subcutaneous adipose tissue (SAT) and visceral adipose tissue (VAT) derived from the same obese individuals [27]. Gene expression has rarely been analyzed between obese and lean ATs even in model organisms such as mice, where a few studies have pursued identification of DEGs between mice fed normal chow and mice fed a high-fat diet (HFD) [28]. Most of these transcriptome- 
based studies have simply concluded that inflammatory genes in ATs are decreased as organisms lose weight and increased as organisms become obese, without asking which cell type in AT was responsible for the increase/decrease in inflammatory genes during weight gain or loss.

It seems that two independent questions are often intermingled in the studies related to obesity and obesity-associated genes described above, (1) whether increased inflammation is involved in fat accumulation and (2) whether increased inflammation is associated with the development of pathogenicity in obese patients. In fact, it is not simple to study relationships between obesity-associated gene expressions and pathogenicity because obesity is not necessarily connected to pathogenic diseases. Here, we investigate gene expression of obese AC samples in comparison with that of lean AC samples, particularly for the expression of inflammatory genes, in order to answer the question \#(1). Further, we also investigate genes that are altered between lean ACs and lean preACs and between obese ACs and obese preACs. Transcriptome-based analysis was conducted using mRNA-Seq data generated from highly purified ACs and preACs obtained from visceral (omental) AT (i.e., VAT) collected during resection surgery from cancer patients.

\section{Results}

\section{Four-way Identification Of Degs}

The flowchart of our analysis is depicted in Fig. 1. After production of mRNA-seq data from the purified ACs and preACs of lean and obese individuals, we investigated two different questions: 1) which genes are significantly upregulated or downregulated under obese conditions in comparison with lean conditions, and 2) which alterations in gene expression detected for obese AC differentiation (i.e., the information extracted from the DEGs obtained by comparing ACs and preACs from obese individuals) are significantly different from those detected for lean AC differentiation (i.e., the information extracted from the DEGs obtained by comparing ACs and preACs from lean individuals). As a validation, we compared our conclusion with that of a previously published paper that provided a list of DEGs obtained by comparing gene expression between lean and obese ACs derived from lean and obese SAT samples; the original dataset produced by the microarray platform was downloaded from the GEO database (GSE80654). How similar that list of DEGs is to our results discussed later in the DISCUSSION section.

We decided to estimate four different classes of DEGs from four different types of the four mRNA-Seq data, i.e., 'lean $A C$ ' (L-AC), 'obese AC' (O-AC), 'lean preAC' (L-preAC), and 'obese preAC' (O-preAC) (see Materials and methods) (Additional file 1: Figure S1): 'Class I: AC-DEGs' from the comparison of expression between 'L-AC' and 'O-AC', 'Class II: preAC-DEGs' between 'L-preAC' and 'O-preAC', 'Class III: Lean_Ag-DEGs' between 'L-preAC' and 'L-AC', and 'Class IV: Obese_Ag-DEGs' between 'O-preAC' and 'O-AC'. Various thresholds were tested to select DEGs (Additional file 2: Table S1), and DEGs were identified for the four classes mentioned above. Class I and II DEGs were investigated to answer question \#1 described above, i.e., to determine the differences between obese ACs and lean ACs and between obese preACs and 
lean preACs. Class III and IV DEGs were chosen to answer question \#2 described above, i.e., to determine how gene expression is altered during obese and lean AC differentiation.

\section{Defining Intermediate Obesity Samples In 'class I: Ac-degs'}

A total of 1,198 genes and 314 genes were classified as 'Class I: AC-DEGs', with $P<0.01$ and $Q<0.05$, respectively (Additional file 2: Table S1). 'L-AC' samples could not be differentiated from 'O-AC' samples by either of these DEGs in the analysis of unsupervised clustering processed with a heatmap; the samples in the middle of the heatmap did not show gene expression patterns pertinent to the 'L-AC' and 'O-AC' categories (Fig. 2A). Notably, Gene ontology (GO) analysis showed that canonical obesity-related genes involved in inflammation and ECM were downregulated in obese ACs rather than in lean ACs (Fig. 2B). The ambiguity of sample classification by these DEGs was also confirmed in principal component analysis (PCA) (Fig. 2C). Thus, we subcategorized these ambiguous samples separately into the 'intermediate (I-AC)' group (\#9, 24, 30, 35, 36, 40, 42, 51). The remaining two extreme samples were then named lean-extreme (' $\mathrm{L}-\mathrm{e}-\mathrm{AC}$ ') and obese-extreme (' $\mathrm{O}_{\mathrm{e}}-\mathrm{AC}$ '), which were ultimately categorized as 8 ' $\mathrm{L}_{\mathrm{e}}-\mathrm{AC}$ ', 8 'I-AC', and 7 ' $\mathrm{O} \mathrm{e}^{-}-\mathrm{AC}$ ', as indicated on top of the heatmap (Additional file 3: Table S2).

Subsequently, for these redefined three groups of samples, some obesity-related clinical information was investigated, including BMI, waist circumference (WC), fasting plasma glucose (FPG) level, C-peptide (Cpep), high-density lipoprotein (HDL) level, and low-density lipoprotein (LDL) level (Fig. 2D). Interestingly, 'I$A C$ ' samples were special in that the BMls of 'I-AC' were expectedly positioned between ' $\mathrm{L}_{\mathrm{e}}-\mathrm{AC}$ ' and ' $\mathrm{O}_{\mathrm{e}}-\mathrm{AC}$ '; however, the WCs and FPGs of 'I-AC' were comparable to those of ' $\mathrm{L}_{e}-\mathrm{AC}$ '. In addition, except for BMI, WC, and FPG, all the other clinical levels showed no significant difference among these three groups, although 'I-AC' was located between ' $\mathrm{L}_{\mathrm{e}}-\mathrm{AC}$ ' and ' $\mathrm{O}_{\mathrm{e}}-\mathrm{AC}$ '. Notably, FPG levels seemed to be associated with WC rather than with BMI.

The existence of a third group, 'I-AC' samples, may not be surprising, considering the complexities of molecular etiologies causing obesity involved with various genetic and epigenetic alterations and the unclear association between obesity and obesity-related metabolic diseases.

\section{Inflammatory genes are expressed at lower levels in obese ACs than in lean ACs}

DEGs were re-estimated involving the 'I-AC' in three different comparison sets, between ' $\mathrm{L}_{\mathrm{e}}-\mathrm{AC}$ ' and ' $\mathrm{O}_{\mathrm{e}}-\mathrm{AC}$ ' (named 'LO-DEGs'), between ' $\mathrm{L}_{\mathrm{e}}-\mathrm{AC}$ ' and 'I-AC' (named 'LI-DEGs'), and between 'I-AC' and ' $\mathrm{O}_{\mathrm{e}}-\mathrm{AC}$ ' (named 'IO-DEGs'), with various thresholds (Additional file 4: Table S3). To understand alterations in gene expression related to obesity, we applied different thresholds to produce similar numbers of DEGs for these three categories (indicated ' $*$ ' in Additional file 4: Table S3). Consequently, a total of 2,657 ( $Q<$ $0.01), 1,474(P<0.01)$, and 1,324 ( $Q<0.05)$ DEGs were selected for 'LO-DEGs', 'LI-DEGs', and 'IO-DEGs', 
respectively; the heatmap of each group was constructed to visualize gene expression differences (Fig. 3A). Notably, the highest number of genes was allocated in 'LO-DEGs', despite the stringent threshold applied. Note that we focused on collecting similar numbers of DEGs rather than on determining a single criterion or a single most important gene (although all the thresholds were chosen in ranges considered statistically significant) to reveal trends in gene expressions between two different conditions.

A striking observation emerged from GO analysis. Specifically, a total of 1,874 genes of the 2,657 'LODEGs' (70.5\%), i.e., genes largely assigned to the inflammatory response, cell adhesion were expressed at significantly lower levels in 'O-AC' than in 'L-AC' (Fig. 3B, in the wide blue box). A similar result was observed in Fig. 2B, and the downregulation of these genes seems to be amplified when 'I-AC' samples are excluded in the DEG analysis. Moreover, 'IO-DEGs' revealed the same pattern as did 'LO-DEGs' (Fig. 3B, in the wide blue box). This observation is striking because these classes of genes are all canonical obesity-related genes that are well-known to be expressed at higher levels in obese AT [19,20].

By contrast, other DEGs involved in fat metabolism in 'LO-DEGs' and 'IO-DEGs' were consistent with the previous findings, i.e., upregulation under obese conditions. For instance, a total of 783 genes of the 2,657 'LO-DEGs' (29.5\%), including LEP, CES1, and NQ01, that were expressed at higher levels in ' $\mathrm{O}_{\mathrm{e}}-\mathrm{AC}$ ' than in ' $\mathrm{L} e-A C$ ', were largely assigned to mitochondrial metabolism and the oxidation-reduction process (ROS) (Fig. 3B, in the wide red box).

'LI-DEGs' were also assigned to distinctive GO functions; RNA metabolism genes were expressed at lower levels in 'I-AC' than in ' $\mathrm{L} e-A C$ ' (i.e., downregulated; narrow blue box in Fig. 3B), and genes involved in centrosome organization and protein phosphorylation were expressed at higher levels in 'I-AC' than in ' $\mathrm{L}_{\mathrm{e}}$ $A C$ ' (i.e., upregulated; narrow red box in Fig. $3 \mathrm{~B}$ ), confirming that 'I-AC' is distinct and not comparable to ' $\mathrm{L}_{\mathrm{e}}-\mathrm{AC}$ ' or ' $\mathrm{O}_{\mathrm{e}}-\mathrm{AC}$ '.

Gene set enrichment analysis (GSEA), i.e., a tool designed to see whether an a priori defined set of genes shows significant differences in expression between two different biological conditions, led to the same conclusion as did GO analysis. Specifically, genes belonging to inflammatory or angiogenesis functions were significantly upregulated in lean ACs rather than obese ACs, whereas genes belonging to cellular respiration functions were significantly upregulated in obese ACs (Additional file 5: Figure S2).

\section{Detailed examination of expression alterations between lean and obese ACs}

The regrouped ' $\mathrm{L}_{\mathrm{e}}-\mathrm{AC}$ ', 'I-AC', and ' $\mathrm{O}_{\mathrm{e}}-\mathrm{AC}$ ' were assumed to reflect different degrees of obesity based on $\mathrm{BMI}$, as shown in Fig. 2D. Thus, changes in gene expression can be examined further by investigating 'LIDEGs' and 'IO-DEGs'. For instance, if a gene that is upregulated both in 'LI-DEGs (i.e., genes expressed at higher levels in 'I-AC' than in ' $\mathrm{L}_{\mathrm{e}}-\mathrm{AC}$ ') and 'IO-DEGs (i.e., genes expressed at higher levels in ' $\mathrm{O}_{\mathrm{e}}{ }^{-\mathrm{AC}}$ ' than in 'I-AC'), it can be concluded that the gene is 'progressively upregulated (named 'progressive-up') because it 
is upregulated in ' $\mathrm{L}_{e}$ ' compared with that in 'I' and upregulated again in 'I' compared with that in ' $\mathrm{O}_{\mathrm{e}}$ ' (refer to Additional file 6: Figure S3). Similarly, if a gene is downregulated in both 'LI-DEGs' and 'IO-DEGs', the gene is defined as progressively downregulated (named 'progressive-down'). Using this scheme, genes were allocated into eight different categories as shown in Fig. 4 and Additional file 6: Figure S3. As a result, only seven genes, including ACAA1, KLHL22, and AKR1C3, were identified as 'progressive-up', while a total of 66 genes were categorized as 'progressive-down'. Notably, genes assigned to cell migration, cell adhesion, and angiogenesis were allocated to the 'progressive-down' category.

Cell cycle and metabolic process genes were upregulated in 'I-AC' compared with those in 'L-AC' and sustained their expression in 'O-AC', i.e., upregulated genes in the 'LI-DEGs' category but not in the 'IODEGs' category (named 'initial-up'). By contrast, RNA processing, angiogenesis, and signal transduction genes were downregulated in 'I-AC' and sustained their expression in 'O-AC', i.e., downregulated genes in the 'LI-DEGs' category but not in the 'IO-DEGs' category (named 'initial-down') (Fig. 4). This result indicates that angiogenesis alteration and cell proliferation may start in the early stage of obesity. Genes involved in ROS metabolism and fatty acid biosynthetic process genes were upregulated in the later stage in obesity, i.e., genes not in the 'LI-DEGs' category but upregulated in the 'IO-DEGs' category (named 'laterup'). By contrast, genes involved in inflammation, cell adhesion, and ECM organization were downregulated in a later stage in obesity, i.e., genes not in the 'LI-DEGs' category but downregulated in the 'IO-DEGs' category (named 'later-down') (Fig. 4). Notably, genes in the 'later-down' category showed that most canonical obesity genes were downregulated in in the later stage of obesity.

\section{Integration Of Degs Between 'ac-degs' And 'preac-degs'}

Next, we investigated similarities and differences between 'Class I: AC-DEGs' and 'Class II: preAC-DEGs' by constructing a graph integrating the two classes of DEGs (Fig. 6). Note that 'LO-DEGs' were used here for the intersecting procedure with 'preAC-DEGs'. Genes were divided into five groups depicted in the graph as blue (representing downregulation in obese samples) or pink (representing upregulation in obese samples) dots connected either to ACs or preACs: 'AC-specific-up', 'AC-specific-down', 'preAC-specific-up', 'preAC-specific-down', and 'Common'.

Two conclusions have been made from this analysis: 1) most DEGs were assigned to the 'AC-' or 'preACspecific' categories, whereas only a few DEGs overlapped with the 'Common' category, indicating that alterations in gene expressions are distinctive for ACs and preACs; 2 ) alterations in gene expression reflected in 'AC-DEGs' were largely in the opposite direction of those in 'preAC-DEGs', as noted above. Again, the inverse relationship of alterations in gene expression was particularly prominent for genes involved in the inflammatory response (Fig. 6). Inflammatory genes were expressed at lower levels in obese ACs (i.e., 'AC-specific-down' category), but they were expressed at higher levels in obese preACs (i.e., 'preAC-specific-up').

Additionally, genes involved in ROS were upregulated in obese ACs, as assigned to the 'AC-specific-up' category. A few DEGs were 'Common' but expressed in the opposite directions between 'AC-DEGs' and 
'preAC-DEGs', depicted as the two-colored balls in the middle of the graph (Fig. 6).

We further investigated whether these DEGs were significantly more enriched with obesity-associated genes than were non-DEGs by mapping them to the obesity-associated genes identified by genome-wide association studies (GWAS). We collected a total of 614 obesity-associated genes from GWASdb2 (http://jjwanglab.org/gwasdb) [30] and intersected them with the DEGs as specifically depicted gene symbols in the graph (Fig. 6). As expected, obesity-associated genes were significantly enriched in 'ACspecific-down' $(P<0.05)$ and 'preAC-specific-down' $(P<0.05)$ (Additional file 8: Table S4). This result may confirm the importance of the functional roles of these DEGs in understanding the molecular alterations leading to obesity.

\section{Both lean and obese AC differentiation are required for the enhancement of inflammatory genes}

Comparing expression between preACs and ACs was assumed to reveal the changes in gene expression during $A C$ differentiation from preAC. Under this assumption, we investigated how obese AC differentiation is differentiated from lean AC differentiation by obtaining Class III and Class IV DEGs; Class III: Lean_Ag-DEGs were estimated by comparing 'L-preAC' and ' $\mathrm{L} e-\mathrm{AC}$ '; and Class IV: Obese_Ag-DEGs were obtained by comparing ' $\mathrm{O}-$ pre $A C^{\prime}$ ' and ' $\mathrm{O} \mathrm{e}_{\mathrm{e}}-\mathrm{AC}$ ' (Additional file 1: Figure $\mathrm{S} 1$ and Additional file 2: Table S1). For the two classes, 'upregulation' or 'downregulation' was determined based on the expression between ACs and preACs, i.e., genes that were expressed at higher levels in ACs than in preACs were upregulated genes, and genes that were expressed at lower levels in ACs than in preACs were downregulated genes.

First, the degree of alterations in gene expression between preACs and ACs was extremely large, regardless of lean or obese samples; a total of 8,448 genes and 10,234 genes were identified as 'Class III: Lean_Ag-DEGs' and 'Class IV: Obese_Ag-DEGs', respectively at Q $<0.01$. Thus, we subcategorized these DEGs into four subcategories by considering the $\log _{2}$ fold change in gene expression along with the $Q<$ 0.01 threshold (Fig. 7). Subsequently, for each of these categories, Class III and Class IV intersected, which led to three subcategories of DEGs, i.e., 'lean AC differentiation-specific (LS)', 'obese AC differentiation-specific (OS)', and 'commonly altered for both (CA)', to investigate how lean AC differentiation and obese $\mathrm{AC}$ differentiation are different from each other.

As a result, most inflammatory genes, such as leukocyte migration, cell chemotaxis, and complement activation genes, were allocated in the highest upregulation category $\left(Q<0.01\right.$ and $\| \log _{2}$ fold change $\left.>4\right)$ for both lean and obese AC differentiation ('CA') (genes in pink boxes in Fig. 7A). However, the extent of upregulation of these inflammatory genes in 'LS' was significantly higher than that of those in 'OS' (Fig. 7B), indicating that both lean and obese AC differentiation are coupled with increased expression of inflammatory genes and that lean $\mathrm{AC}$ differentiation, rather than obese $\mathrm{AC}$ differentiation, requires stronger upregulation of these inflammatory genes. 
In addition, RNA metabolism genes were downregulated for both 'LS' and 'OS' (Fig. 7A, genes boxed in yellow) but with small fold changes, indicating that both lean and obese AC differentiation are required for dynamic alterations in gene expression machineries. Interestingly, genes involved in mitochondrial metabolism, including oxidative phosphorylation or electron transport, were upregulated with small fold changes in either 'LS' or 'OS', but no genes were altered in the 'CA' category (Fig. 7A, genes in green boxes), indicating that different kinds of mitochondrial metabolism genes are slightly but significantly altered during lean and obese AC differentiation. In contrast, fatty acid metabolism and catabolic process genes were mostly allocated to the 'CA' category with a wide range of fold changes (Fig. 7A, genes boxed gray), indicating that some common metabolic processes underlie both lean and obese AC differentiation.

\section{Discussion}

Obesity is often characterized as a low-grade inflammatory disease in which an enhanced inflammatory response in AT and the serum of obese organisms has been well established [31]. However, surprisingly, studies that addressed the possible enhancement of inflammatory genes by analyzing DEGs between obese fats and lean fats collected from obese and lean cohorts are extremely rare, which is primarily due to the difficulties of obtaining fat samples derived from lean and healthy individuals.

By estimating DEGs between lean ATs and obese ATs respectively derived from the human lean and obese cohorts, we reached a contradictory conclusion regarding the up- or downregulation of inflammatory genes involved in obesity. We found that inflammatory genes such as IL-6 and TNF-a were actually expressed at lower levels in obese ACs than in lean ACs and at higher levels in obese preACs than in lean preACs, indicating that 1) inflammatory genes are downregulated in obese conditions for ACs but 2) upregulated in obese conditions for preACs. The downregulation of these inflammatory genes, including IL-6 and TNF-a, were partly confirmed by intersecting the list of our DEGs and the DEGs that were previously published [32] and deposited in the GEO database (GSE80654), as will be discussed below in detail.

These results led us to revisit the notion that the upregulation of inflammatory genes may be the primary cause of obesity. We thus designed another comparison of gene expression between ACs and preACs, i.e., a comparison that was expected to estimate gene expression differences that occur during AC differentiation. As shown in Fig. 7, we showed that ACs express higher levels of inflammatory genes than do preACs for both lean and obese conditions, indicating that both lean and obese AC differentiation require increased inflammatory response genes. Interestingly, we observed that the extent of enhancement of inflammatory genes in ACs compared with preACs was significantly higher for lean conditions than for obese conditions (Fig. 7A and B). We think that this result clearly supports Asterholm et al.'s (2014) view of the positive role of inflammation in fat deposition, i.e., an attenuated inflammatory response may be linked to more harmful fat deposition. 
We validated our work by comparing with similar previous studies that were conducted based on an experimental design similar to that in the present work. Unfortunately, only one paper was found where the authors reported DEGs that were significantly altered in obese ACs compared with lean ACs purified from human AT samples [32]; in total, 24 upregulated DEGs and 64 downregulated DEGs were reported. Note that the ACs in this study were derived from SAT rather than VAT. Interestingly, we found significant overlap in the list of 'LO-DEGs' between our study and Ehrlund et al.'s study. For instance, genes, including NQ01, VLDLR, etc., were upregulated, whereas genes including IL-6, MMP2, and CD44 (i.e., inflammatory response genes) were downregulated in obese ACs compared with those in lean ACs. Approximately $21.6 \%$ (19/88) overlapped with the same direction (i.e., up or down) between our study and Ehrlund et al.'s study. We think that this degree of overlap is quite remarkable, considering that we used an mRNA-Seq platform whereas they used a microarray platform to produce transcriptome data, and that a significant gene expression difference has been reported between SAT and VAT [33, 34]. In addition, Xing et al. (2015)'s RNA-Seq-based transcriptome study of pigs showed that pigs with thicker back fats (corresponding to obese ATs in the present work) showed a significant lower expression of inflammatory genes than pigs with thinner back fats (corresponding to lean ATs in the present work), which is consistent with our observation in the present work that inflammatory genes are downregulated in obese ACs compared to lean ACs.

Another important but often forgotten aspect is that AT is a complex organ with a residing mixture of highly heterogeneous cell types, including macrophages, other immune cells, preACs, endothelial cells, and lipid-filled ACs [35-38]. Compositional changes in these cells are associated with obesity and construct unique microenvironments within AT, entailing the synthesis and turnover of ECM components that lead to changes in adiposity accompanying limited/excessive nutritional supply [39]. A key player in regulating AT inflammation is macrophage inflammation. The involvement of macrophages in AT associated with obesity is known as M1 macrophage polarization. Unfortunately, due to the lack of purified macrophages, we were unable to investigate whether macrophages have a major role in enhanced inflammation in obese AT. Several studies have already noted that the source of enhanced inflammatory cytokines such as TNF- $a$ and IL-6 is macrophages rather than ACs [40, 41]. Instead, in the present work, we suggest that preACs are partly responsible for the enhanced inflammatory responses in obese AT.

\section{Conclusions}

Using transcriptome-based analyses of mRNA-Seq data derived from human purified ACs and preACs between lean and obese conditions, we found that the canonical obesity-related upregulated genes, particularly inflammatory response genes, were expressed at significantly lower levels in obese ACs than in lean ACs. Moreover, the levels of these classes of genes increased in both lean ACs and obese ACs compared to the respective lean preACs and obese preACs; however, the levels of enhancement of these genes were even greater for lean ACs than obese ACs. 
To our knowledge, our study is the first to investigate alterations in gene expression using the human transcriptome from purified preACs and ACs in lean and obese VAT. We believe our present work will help to resolve some of the unanswered questions regarding the molecular alterations that occur in lean and obese fat accumulation.

\section{Methods}

\section{Preparation of transcriptomes derived from human $A C$ and preAC samples}

All the transcriptome samples used in the present work were produced by one of the out-sourced studies performed by the Korea National Institute of Health $(\mathrm{KNIH})$. KNIH made efforts to collecting epigenomes and transcriptomes as a participating institute of the International Human Epigenome Consortium (IHEC), granting research funds to recruited research groups (selected by an evaluation process) for collecting tissue samples and their epigenome data. The collected data were also strictly regulated and distributed by the $\mathrm{KNIH}$ to the research groups who had proposed to analyze them after evaluation. We were one of the research groups that were selected to access and analyze the raw data in the KNIH server called the open access (OA) system under limited permission.

\section{Ethics Statement}

This study was performed in accordance with the principles of the Declaration of Helsinki and was approved by the Kangwon National University Hospital (Chuncheon, Korea) Institutional Review Board (IRB) (KWNUIRB-2017-11-003).

\section{Purification Of Ac And Preac Samples}

Retrieving VAT from healthy individuals is extremely difficult, and purification of ACs or preACs from the small amount of lean VAT is even more difficult, so that we purified ACs and preACs from AT that was isolated from VAT in the abdominal region during surgical resection of human cancer patients. To exclude a possibility that gene expression in AT can be affected by tumors residing in locations distant from the AT we collected, we first confirmed that there was no cancer type bias between lean and obese samples (Additional file 9: Table S5). Second, we confirmed that the CRP levels, i.e., an indicator of systemic inflammation, of the samples that we analyzed were all less than 1 except one for 1 outlier (\#ob50), indicating that no systemic cachexia response affected gene expression for both lean and obese ATs (or ACs/preACs) from the tumors (Additional file 10: Table S6). In addition, we found no bias in sex, age, cancer grades between lean and obese samples (Additional file 10: Table S6).

Then, AT with blood vessels and connective tissue removed was washed with PBS to remove blood, including white and red blood cells. The $100 \sim 200 \mathrm{~g}$ of fat that was collected from each patient was 
minced and treated with collagenase I for 1 hour at $37^{\circ} \mathrm{C}$, in which the samples were washed three times with PBS every 20 minutes. The digested samples were filtered with $350 \mu \mathrm{m}$ mesh to remove undigested tissue. Fetal bovine serum (10\%) was added to stop the collagenase I reaction.

After centrifugation at $400 \mathrm{xg}$ for 10 minutes, the supernatant fraction, i.e., the fraction containing mature ACs, and the stromal vascular fraction (SVF) pellet were collected separately (Additional file 11: Figure S5). Mature ACs washed with PBS and medium were then used for RNA isolation. The SVF pellet was incubated in red blood cell lysis buffer for 15 minutes to remove red blood cells, and the SVF pellet was retrieved again after centrifugation. The cells in the SVF pellet were filtered through $100 \mu \mathrm{m}$ mesh, and $40 \mu \mathrm{m}$ nylon mesh was used for MACS/FACS sorting to purify preadipocytes (CD45-/CD34+/CD31 cells) (Additional file 11: Figure S5) [42].

\section{Rna Extraction}

We tried to collect ACs and preACs as a pair of samples obtained from the ATs from the same individual, but the success rate of extracting preACs was not very high, which is why the number of preACs is significantly lower than that of ACs, as described below.

Total RNA from 27 AC and 21 preAC samples was extracted with an RNeasy Lipid tissue kit (Qiagen, Hilden, Germany) and RNeasy Micro Kit (Qiagen, Hilden, Germany) using the manufacturer's recommendations. Samples from AC comprising $12 \mathrm{~L}-\mathrm{AC}$ and 15 O-AC samples, whereas preAC samples consisted of $3 \mathrm{~L}$-preACs and 18 O-preACs. Obesity among collected patient samples was diagnosed by $\mathrm{BMI}>25 \mathrm{~kg} / \mathrm{m}^{2}$ rather than $\mathrm{BMI}>30 \mathrm{~kg} / \mathrm{m}^{2}$. Based on a report from the Korea National Institute of Health, Koreans are particularly troubled by a higher incidence of metabolic diseases coupled with obesity, even though they have a BMI lower than the worldwide average. This is why Korean obesity is diagnosed as $\mathrm{BMl}>25 \mathrm{~kg} / \mathrm{m}_{2}$ rather than $\mathrm{BMI}>30 \mathrm{~kg} / \mathrm{m}_{2}[43,44]$.

\section{Cdna Library Preparation}

Construction of cDNA libraries and sequencing were conducted by two different protocols for the two different batches of samples collected at different times. First, for the samples, including L-AC samples (\#5, 9, 29, 34, 35, 37, 38, 39, 40, 42, 43, 46), O-AC samples (\#3, 11, 24, 25, 30, 32, 36, 41, 49, 50, 51), LpreAC samples $(\# 2,10,13)$, and 0-preAC samples $(\# 4,8,12,18,19,20,21,33,53,54)$, cDNA libraries were constructed by a TruSeq ${ }^{\circledR}$ RNA Sample Preparation Guide (Illumina, San Diego, CA) with the purified RNAs. rRNAs were removed using a Ribo-zero-rRNA removal kit (Illumina). Quality control (QC) of samples was then conducted with a Bioanalyzer (Agilent Technologies, Santa Clara, CA). Sequencing of each clone in the constructed cDNA library was performed with the HiSeq 2000 platform (Illumina, San Diego, CA). Second, for the samples including L-AC (\#59, 62, 64, 65) and 0-preAC (\#55, 56, 57, 58, 60, 61, $63,66)$, cDNA libraries were constructed by using a TruSeq Stranded mRNA Sample Preparation Guide. After sample QC using the Bioanalyzer was performed as for the first sample groups, sequencing of each 
clone was conducted with the Hiseq2500 platform. Unfortunately, different cDNA libraries constructed by these two different protocols severely affected the classification of samples, so we decided to exclude all the cDNAs constructed at later stages from further analyses.

\section{Mrna-seq Analysis To Obtain Degs}

The sequencing reads of the samples mentioned above were checked for quality by FastQC (https://www.bioinformatics.babraham.ac.uk/). The adaptor sequences and low-quality sequences were trimmed by Trimmomatic (v0.35) [45]. The reference genome fasta file (GRCh38/hg38) was indexed by STAR (v2.7.1a) using the 'genomeGenerated' option. Then, the trimmed reads were mapped using STAR (v2.7.1a) to the indexed reference genome [46]. Annotation was conducted using the 'GTF' file of 'GENCODE Gene Set' (release 30) (https://www.gencodegenes.org/). Mapped reads were quantified using 'htseq-count'. The read counts were then normalized and compared between the control and case samples (e.g., 'L-AC vs. O-AC', 'L-preAC vs. O-preAC', etc.) using 'DESeq2' [47].

\section{Data analysis}

All statistical analyses and plots were performed using R (v3.5.1) [48] with the Bioconductor package (v3.8) [49]. GO analysis was performed using the DAVID tool [50]. GSEA was performed using GSEA software (v3.0) [51]. Furthermore, we used the 'clusterprofiler' package and 'ggplot2' function in R (v3.5.1) for additional GO analysis and plotting [52]. The network graph of genes was constructed by using Cytoscape software (v3.7.1) [53]. Other batch jobs were performed with custom-built Python scripts (v3.6.8) (https://www.python.org/).

\section{Availability Of Data And Materials}

All RNA-Seq data are accessible on the KNIH OA system through gaining specific permission by the evaluation of $\mathrm{KNIH}$.

\section{Abbreviations}

AT

adipose tissue

AC

adipocyte

preAC

preadipocyte

DEG

differentially expressed gene

BAT 
brown adipose tissue

SAT

subcutaneous white adipose tissue

VAT

visceral white adipose tissue

SVF

stromal vascular fraction

L-AC

lean adipocyte

L-preAC

lean preadipocyte

O-AC

obese adipocyte

O-preAC

obese preadipocyte

QC

quality control

$\mathrm{GO}$

gene ontology

GSEA

gene set enrichment analysis

BMI

body mass index

WC

waist circumference

FBS

fasting blood sugar

C-pep

c-peptide

HDL

high-density lipoprotein

LDL

low-density lipoprotein

LS

lean adipogenesis-specific

OS

obese adipogenesis-specific

CA

commonly altered for both

DM 
diabetes mellitus

HTN

hypertension

\section{Declarations}

\section{Acknowledgements}

Not applicable.

\section{Funding}

This research was supported by the Basic Science Research Program through the National Research Foundation of Korea (NRF) funded by the Ministry of Education, Science and Technology (NRF2019R1A2C1002350) and by an intramural grant from the Korea National Institute of Health (2017NI73002-02). This study was performed with biospecimens and data provided by the Reference Data Production of Regulation of Disease Gene Expression (4848-308), which is supported by the Korea Centers for Disease Control and Prevention, Republic of Korea.

\section{Authors' contributions}

S.S. Choi conceived and designed the experiments; S.H. Lee and N.H. Choi performed data analysis; I.U. Koh, N.H. Choi, B.J. Kim, S. Lee and S.C. Kim contributed reagents and materials. S.S. Choi and S.H. Lee wrote the paper. All authors have read and approved the manuscript.

\section{Corresponding author}

Correspondence to Sun Shim Choi.

\section{Ethics approval and consent to participate}

This study was performed in accordance with the principles of the Declaration of Helsinki and was approved by the Kangwon National University Hospital (Chuncheon, Korea) Institutional Review Board (IRB) (KWNUIRB-2017-11-003).

\section{Consent for publication}

Not applicable.

\section{Competing interests}

The authors declare no conflict of interests

\section{References}


1. Ellulu MS, Patimah I, Khaza'ai H, Rahmat A, Abed Y. Obesity and inflammation: the linking mechanism and the complications. Arch Med Sci 2017 Jun;13(4):851-863.

2. Stern JH, Rutkowski JM, Scherer PE. Adiponectin, leptin, and fatty acids in the maintenance of metabolic homeostasis through adipose tissue crosstalk. Cell metabolism 2016;23(5):770-784.

3. Raclot T. Selective mobilization of fatty acids from adipose tissue triacylglycerols. Prog Lipid Res 2003;42(4):257-288.

4. Galic S, Oakhill JS, Steinberg GR. Adipose tissue as an endocrine organ. Mol Cell Endocrinol 2010;316(2):129-139.

5. Heindel JJ, Newbold R, Schug TT. Endocrine disruptors and obesity. Nature Reviews Endocrinology 2015;11(11):653-661.

6. Stolarczyk E. Adipose tissue inflammation in obesity: a metabolic or immune response? Current opinion in pharmacology 2017;37:35-40.

7. Catalan V, Gomez-Ambrosi J, Rodriguez A, Fruehbeck G. Role of extracellular matrix remodelling in adipose tissue pathophysiology. Relevance in the development of obesity. 2012.

8. Sun K, Kusminski CM, Scherer PE. Adipose tissue remodeling and obesity. J Clin Invest 2011 Jun;121(6):2094-2101.

9. Lin D, Chun T, Kang L. Adipose extracellular matrix remodelling in obesity and insulin resistance. Biochem Pharmacol 2016;119:8-16.

10. Ruiz-Ojeda FJ, Méndez-Gutiérrez A, Aguilera CM, Plaza-Díaz J. Extracellular Matrix Remodeling of Adipose Tissue in Obesity and Metabolic Diseases. International Journal of Molecular Sciences 2019;20(19):4888.

11. Han J, Wei L, Xu W, Lu J, Wang C, Bao Y, et al. CTSK inhibitor exert its anti-obesity effects through regulating adipocyte differentiation in high-fat diet induced obese mice. Endocr J 2014:EJ14-0336.

12. Chen L, Lu B, Yang Y, Zhang W, Wang X, Zhou H, et al. Elevated circulating cathepsin S levels are associated with metabolic syndrome in overweight and obese individuals. Diabetes Metab Res 2019;35(3):e3117.

13. Song M, Lee H, Jin B, Gutierrez-Aguilar R, Shin K, Choi S, et al. Depot-specific differences in angiogenic capacity of adipose tissue in differential susceptibility to dietinduced obesity. Molecular metabolism 2016;5(11):1113-1120. 
14. Simon M, Tournaire R, Pouyssegur J. The angiopoietin-2 gene of endothelial cells is upregulated in hypoxia by a HIF binding site located in its first intron and by the central factors GATA-2 and Ets-1. J Cell Physiol 2008;217(3):809-818.

15. Choe SS, Huh JY, Hwang IJ, Kim JI, Kim JB. Adipose tissue remodeling: its role in energy metabolism and metabolic disorders. Frontiers in endocrinology 2016;7:30.

16. Sattar N, Gill JM. Type 2 diabetes as a disease of ectopic fat? BMC medicine 2014;12(1):123.

17. Furukawa S, Fujita T, Shimabukuro M, Iwaki M, Yamada Y, Nakajima Y, et al. Increased oxidative stress in obesity and its impact on metabolic syndrome. J Clin Invest 2004 Dec;114(12):1752-1761.

18. Sproston NR, Ashworth JJ. Role of C-reactive protein at sites of inflammation and infection. Frontiers in immunology 2018;9:754.

19. Saltiel AR, Olefsky JM. Inflammatory mechanisms linking obesity and metabolic disease. J Clin Invest 2017;127(1):1-4.

20. Gregor MF, Hotamisligil GS. Inflammatory mechanisms in obesity. Annu Rev Immunol 2011;29:415-445.

21. Ye J, McGuinness OP. Inflammation during obesity is not all bad: evidence from animal and human studies. American Journal of Physiology-Endocrinology and Metabolism 2012;304(5):E466-E477.

22. Asterholm IW, Tao C, Morley TS, Wang QA, Delgado-Lopez F, Wang ZV, et al. Adipocyte inflammation is essential for healthy adipose tissue expansion and remodeling. Cell metabolism 2014;20(1):103-118.

23. Hotamisligil GS. Inflammation, metaflammation and immunometabolic disorders. Nature 2017;542(7640):177.

24. Rutkowski JM, Stern JH, Scherer PE. The cell biology of fat expansion. J Cell Biol 2015 Mar 2;208(5):501-512.

25. Xing K, Zhu F, Zhai L, Liu H, Wang Y, Wang Z, et al. Integration of transcriptome and whole genomic resequencing data to identify key genes affecting swine fat deposition. PLoS One 2015 Apr 7;10(4):e0122396.

26. Mardinoglu A, Heiker JT, Gärtner D, Björnson E, Schön MR, Flehmig G, et al. Extensive weight loss reveals distinct gene expression changes in human subcutaneous and visceral adipose tissue. Scientific reports 2015;5(1):1-11. 
27. Gerhard GS, Styer AM, Strodel WE, Roesch SL, Yavorek A, Carey DJ, et al. Gene expression profiling in subcutaneous, visceral and epigastric adipose tissues of patients with extreme obesity. Int J Obes 2014;38(3):371-378.

28. Kim H, Ryoo ZY, Choi SU, Lee S. Gene expression profiles reveal effect of a high-fat diet on the development of white and brown adipose tissues. Gene 2015;565(1):15-21.

29. Ortega FJ, Moreno-Navarrete JM, Pardo G, Sabater M, Hummel M, Ferrer A, et al. MiRNA expression profile of human subcutaneous adipose and during adipocyte differentiation. PloS one 2010;5(2):e9022.

30. Li MJ, Liu Z, Wang P, Wong MP, Nelson MR, Kocher JA, et al. GWASdb v2: an update database for human genetic variants identified by genome-wide association studies. Nucleic Acids Res 2015;44(D1):D869-D876.

31. Mraz M, Haluzik M. The role of adipose tissue immune cells in obesity and low-grade inflammation. J Endocrinol 2014;222(3):R113-R127.

32. Ehrlund A, Acosta JR, Björk C, Hedén P, Douagi I, Arner P, et al. The cell-type specific transcriptome in human adipose tissue and influence of obesity on adipocyte progenitors. Scientific data 2017;4:170164.

33. DeClercq VC, Goldsby JS, McMurray DN, Chapkin RS. Distinct adipose depots from mice differentially respond to a high-fat, high-salt diet. J Nutr 2016;146(6):1189-1196.

34. Spoto B, Di Betta E, Mattace-Raso F, Sijbrands E, Vilardi A, Parlongo R, et al. Pro-and anti-inflammatory cytokine gene expression in subcutaneous and visceral fat in severe obesity. Nutrition, Metabolism and Cardiovascular Diseases 2014;24(10):1137-1143.

35. Boutens L, Stienstra R. Adipose tissue macrophages: going off track during obesity. Diabetologia 2016;59(5):879-894.

36. Huh JY, Park YJ, Ham M, Kim JB. Crosstalk between adipocytes and immune cells in adipose tissue inflammation and metabolic dysregulation in obesity. Mol Cells 2014 May;37(5):365-371.

37. Jeffery E, Wing A, Holtrup B, Sebo Z, Kaplan JL, Saavedra-Peña R, et al. The adipose tissue microenvironment regulates depot-specific adipogenesis in obesity. Cell metabolism 2016;24(1):142-150.

38. Ràfols ME. Adipose tissue: cell heterogeneity and functional diversity. Endocrinología y Nutrición (English Edition) 2014;61(2):100-112. 
39. Mariman EC, Wang P. Adipocyte extracellular matrix composition, dynamics and role in obesity. Cellular and molecular life sciences 2010;67(8):1277-1292.

40. Castoldi A, Naffah de Souza C, Câmara NOS, Moraes-Vieira PM. The macrophage switch in obesity development. Frontiers in immunology 2016;6:637.

41. Liu K, Zhao E, Ilyas G, Lalazar G, Lin Y, Haseeb M, et al. Impaired macrophage autophagy increases the immune response in obese mice by promoting proinflammatory macrophage polarization. Autophagy 2015;11(2):271-284.

42. Acosta JR, Douagi I, Andersson DP, Bäckdahl J, Rydén M, Arner P, et al. Increased fat cell size: a major phenotype of subcutaneous white adipose tissue in non-obese individuals with type 2 diabetes. Diabetologia 2016;59(3):560-570.

43. Kim Y, Suh YK, Choi H. BMI and metabolic disorders in South Korean adults: 1998 Korea national health and nutrition survey. Obes Res 2004;12(3):445-453.

44. Kim MK, Lee W, Kang J, Kang J, Kim BT, Kim SM, et al. 2014 clinical practice guidelines for overweight and obesity in Korea. Endocrinology and Metabolism 2014;29(4):405-409.

45. Bolger AM, Lohse M, Usadel B. Trimmomatic: a flexible trimmer for Illumina sequence data. Bioinformatics 2014;30(15):2114-2120.

46. Dobin A, Davis CA, Schlesinger F, Drenkow J, Zaleski C, Jha S, et al. STAR: ultrafast universal RNA-seq aligner. Bioinformatics 2013;29(1):15-21.

47. Love MI, Huber W, Anders S. Moderated estimation of fold change and dispersion for RNA-seq data with DESeq2. Genome Biol 2014;15(12):550.

48. Ripley BD. The R project in statistical computing. MSOR Connections. The newsletter of the LTSN Maths, Stats \& OR Network 2001;1(1):23-25.

49. Huber W, Carey VJ, Gentleman R, Anders S, Carlson M, Carvalho BS, et al. Orchestrating high-throughput genomic analysis with Bioconductor. Nature methods 2015;12(2):115.

50. Huang DW, Sherman BT, Lempicki RA. Bioinformatics enrichment tools: paths toward the comprehensive functional analysis of large gene lists. Nucleic Acids Res 2008;37(1):113.

51. Subramanian A, Tamayo P, Mootha VK, Mukherjee S, Ebert BL, Gillette MA, et al. Gene set enrichment analysis: a knowledge-based approach for interpreting genome-wide expression profiles. Proc Natl Acad Sci U S A 2005 Oct 25;102(43):15545-15550. 
52. Yu G, Wang L, Han Y, He Q. clusterProfiler: an R package for comparing biological themes among gene clusters. Omics: a journal of integrative biology 2012;16(5):284-287.

53. Shannon P, Markiel A, Ozier O, Baliga NS, Wang JT, Ramage D, et al. Cytoscape: a software environment for integrated models of biomolecular interaction networks. Genome Res 2003 Nov;13(11):2498-2504.

\section{Supplementary Files Legend}

\section{Additional files}

Additional file 1: Figure S1. Schematic of identification of four different classes of DEGs.

Class I: AC-DEGs are identified from O-AC expression divided by L-AC expression, Class II: preAC-DEGs are from 0-preAC expression divided by L-preAC expression, Class III: Lean_Ag-DEGs are from L-AC expression divided by L-preAC expression, and Class IV are from O-AC expression divided by O-preAC expression. The statistical criteria for detecting each of the DEGs are explained in Supplementary Table 1. 'e[' indicates the expression levels of genes within the bracket.

\section{Additional file 2: Table S1. Statistical thresholds tested for selecting DEGs.}

Additional file 3: Table S2. The sample information used for the present analysis.

\section{Additional file 4: Table S3. Statistical thresholds used for testing to select three subcategories of AC- DEGs.}

\section{Additional file 5: Figure S2. GSEA analysis of LO-DEGs.}

Four selected gene sets from GSEA analysis are presented here. (A) and (B) are the gene sets for inflammatory response and angiogenesis, showing significant enrichment in $\mathrm{L}_{\mathrm{e}^{\prime}}(C)$ and (D) are the gene sets for cellular respiration and cellular metabolism, showing significant enrichment in $\mathrm{O}_{\mathrm{e}}$. Left panels represent the graph of the enrichment score (ES) generated from GSEA, and the right panels are heatmaps constructed by the gene sets with significant ES scores. Cyan and red bars on top of the heatmaps represent the ' $\mathrm{L}_{\mathrm{e}}-\mathrm{AC}$ ' and ' $\mathrm{O}_{\mathrm{e}}-\mathrm{AC}$ ' samples, respectively.

\section{Additional file 6: Figure S3. Schematic of strategy for dissecting DEGs into eight subcategories.}

'LI-DEGs' and 'IO-DEGs' are intersected. A gene is determined to be 'progressive-up', when the gene that is upregulated both in 'LI-DEGs' and 'IO-DEGs'. A gene is defined as 'progressive-down' when the gene is downregulated both in 'LI-DEGs' and 'IO-DEGs'. A gene is 'initial-up', when the gene is upregulated in 'LIDEGs' but is not in 'IO-DEGs'. A gene is 'initial-down' when the gene is downregulated in 'LI-DEGs' but not in 'IO-DEGs'. A gene is 'later-up' when the gene is not upregulated in 'LI-DEGs' but is upregulated in 'IODEGs'. A gene is 'later-down' when the gene is not downregulated in 'LI-DEGs' but is downregulated in 'IODEGs. A gene is 'up-to-down' when the gene is upregulated in LI-DEGs and downregulated in IO-DEGs and 'down-to-up' when the reverse applies. 
Additional file 7: Figure S4. Principle Component Analysis (PCA) plot of the preAC-DEGs.

The L-preAC (turquoise) and 0-preAC (red) samples are dotted along the axis of first two principal component (PC1 and PC2).

Additional file 8: Table S4. Statistical tests for the enrichment of DEGs in the obesity-related genes identified by GWAS .

Additional file 9: Table S5. Clinical information of samples.

Additional file 10: Table S6. Statistical summary of clinical information.

Additional file 11: Figure S5. Schematic of purification of AC and preAC cells from AT.

Refer to Materials and methods for the detailed procedures depicted in this schematic.

\section{Figures}

Fig. 1

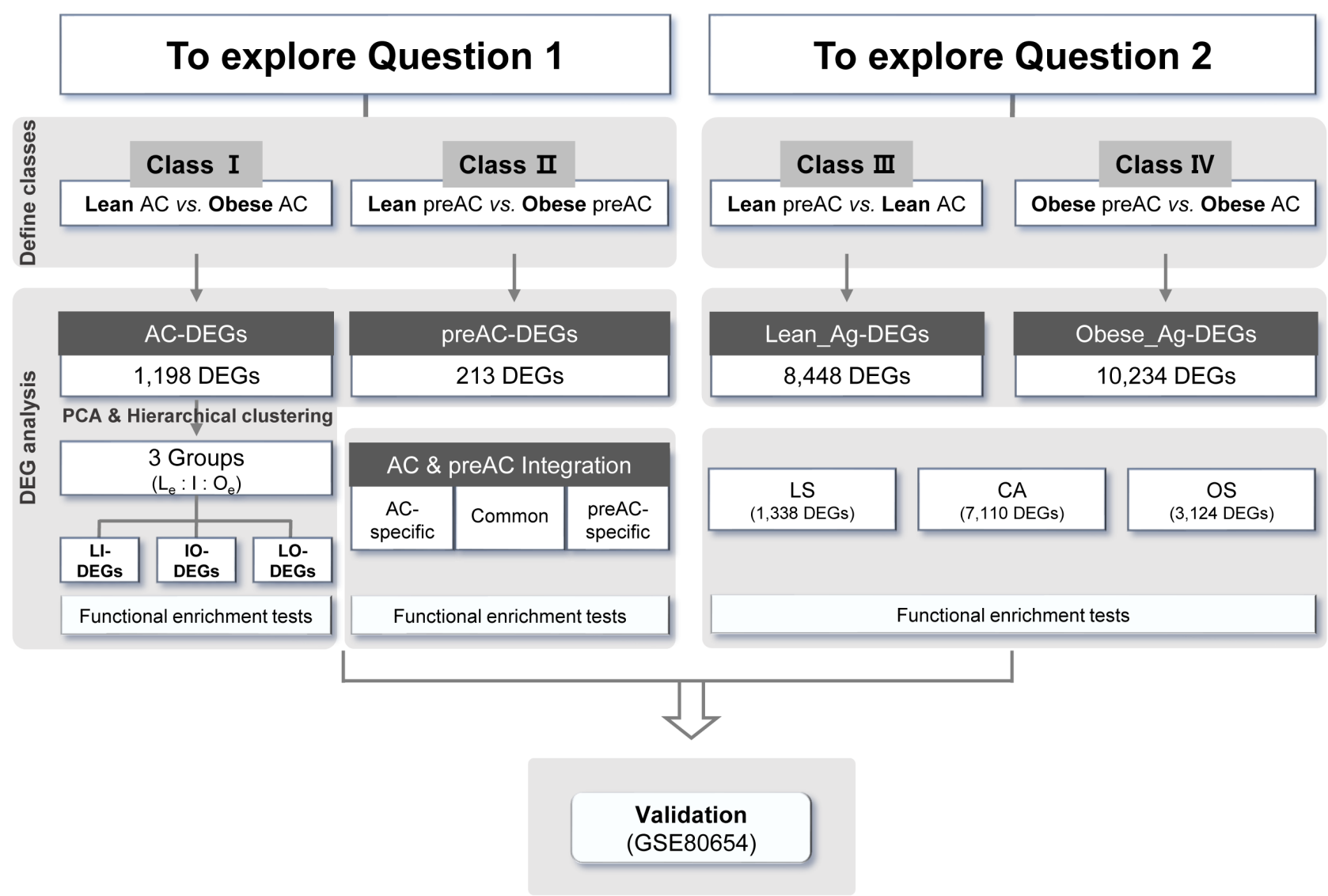


Figure 1

Overall schematic of the workflow. Workflow is depicted as a flowchart. Abbreviations used in this flowchart are as follows; $A C$, adipocyte; preAC, preadipocyte; $D E G$, differentially expressed gene; Le, lean extreme; Oe, obese extreme; Ag, AC differentiation; LS, lean AC differentiation-specific; OS, obese AC differentiation-specific; CA, commonly altered for both.

Fig. 2

A

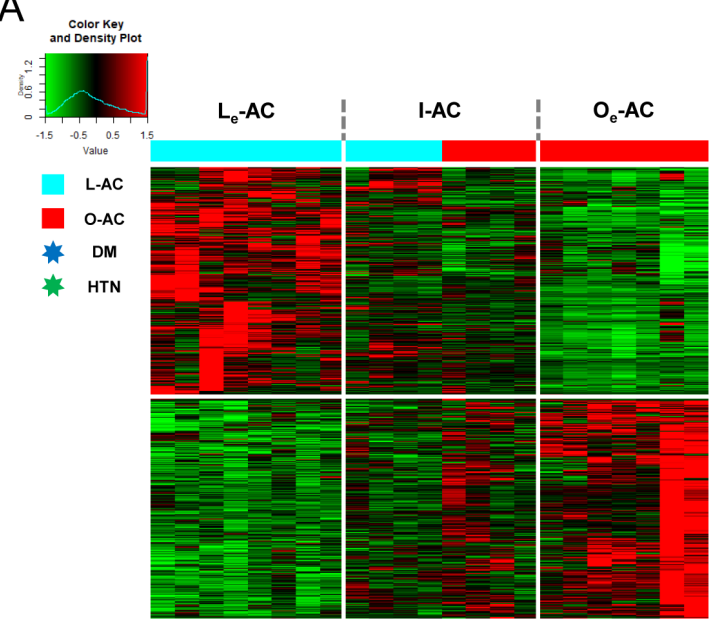

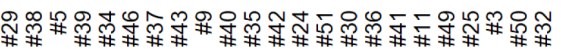

C
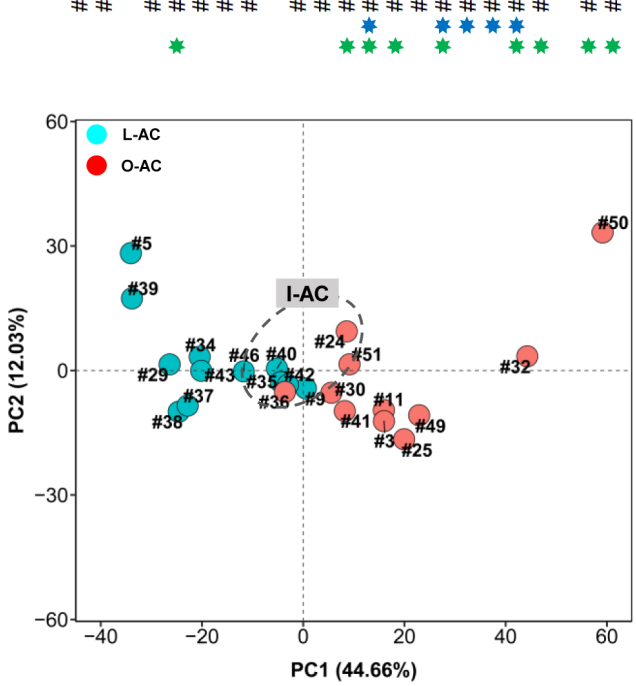

B

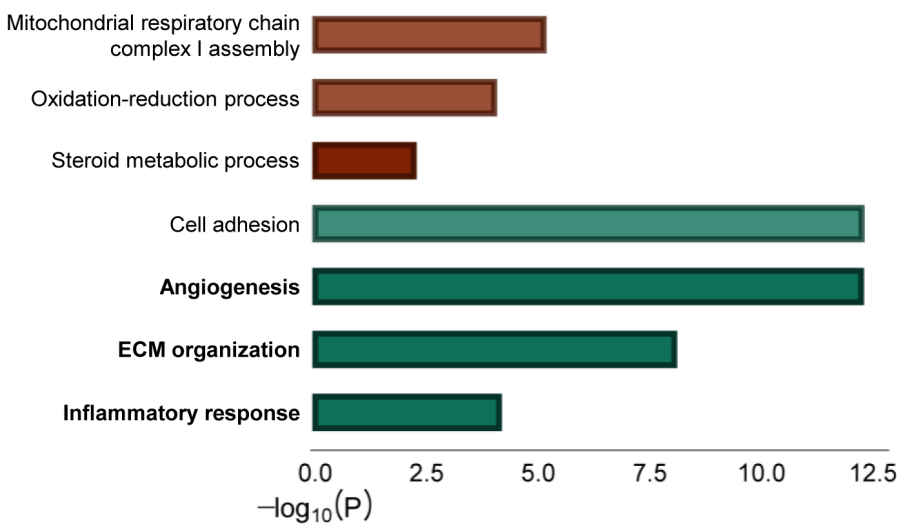

D
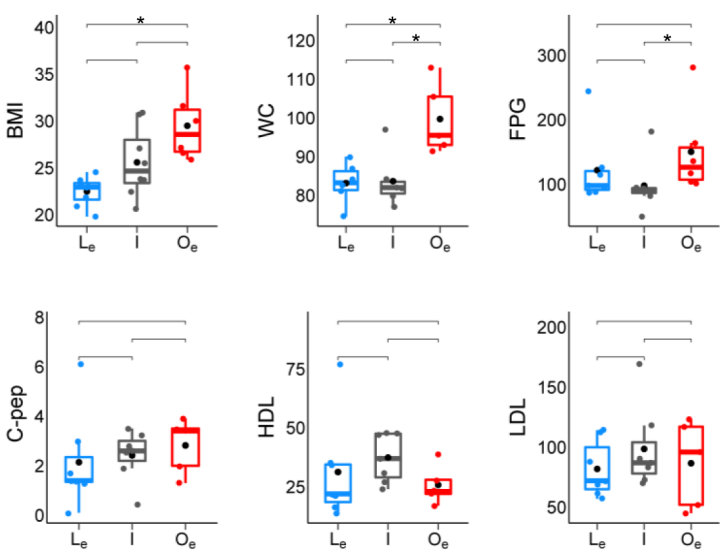

\section{Figure 2}

Classification of AC samples by DEGs. A. Construction of the heatmap accompanied by unsupervised hierarchical clustering. Heatmap coupled with unsupervised hierarchical clustering is generated by the 1,198 DEGs that are estimated by comparing mRNA expression with $P<0.01$ between 12 L-AC and 11 OAC samples (see Materials and methods). Cyan and red bars located at top of the heatmap are replaced for the dendrograms generated by the clustering procedure where cyan and red bars represent lean and obese samples, respectively. 'Le', 'l', and 'Oe' represent extreme, intermediate, and obese extremes, respectively, as explained in the main text. The samples in blue and green ' $*$ ' indicate whether the patients have been treated with drugs for diabetes (DM) or hypertension (HTN), respectively. B. GO analysis of 'AC- 
DEGs'; red and green bars represent upregulated (i.e., genes with higher levels in obese ACs than in lean ACs) and downregulated genes (i.e., genes with lower levels in obese ACs than in lean ACs), respectively. The functional terms with top significance by sorting $P$ values in the $G O$ analysis are represented only in the graph. The scale on the bottom indicates the - $\log 10 P$ value of the significance of enrichment of each functional term. C. PCA analysis. PCA analysis is performed with 'Class I: AC-DEGs' (see Materials and methods). The emergence of 'I-AC' samples identified in (A) is confirmed as the samples in the group are located in the middle of the plot marked with dotted circles. D. Box plot analysis of selected clinical information among 'Le-AC', 'I-AC', and 'Oe-AC' samples; BMI, WC, FPG, C-pep, LDL, and HDL were selected. The statistical test of clinical information among the groups was performed by the 'Wilcoxon rank-sum test'. ' $*$ ' indicates that the statistical test is $\mathrm{P}<0.05$.

Fig. 3

A

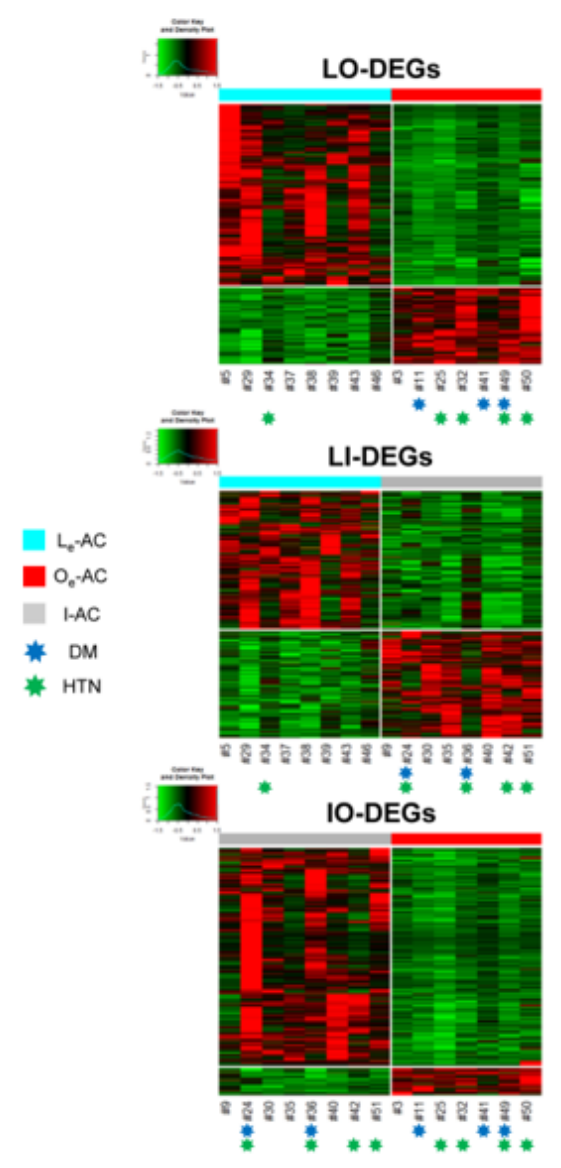

B

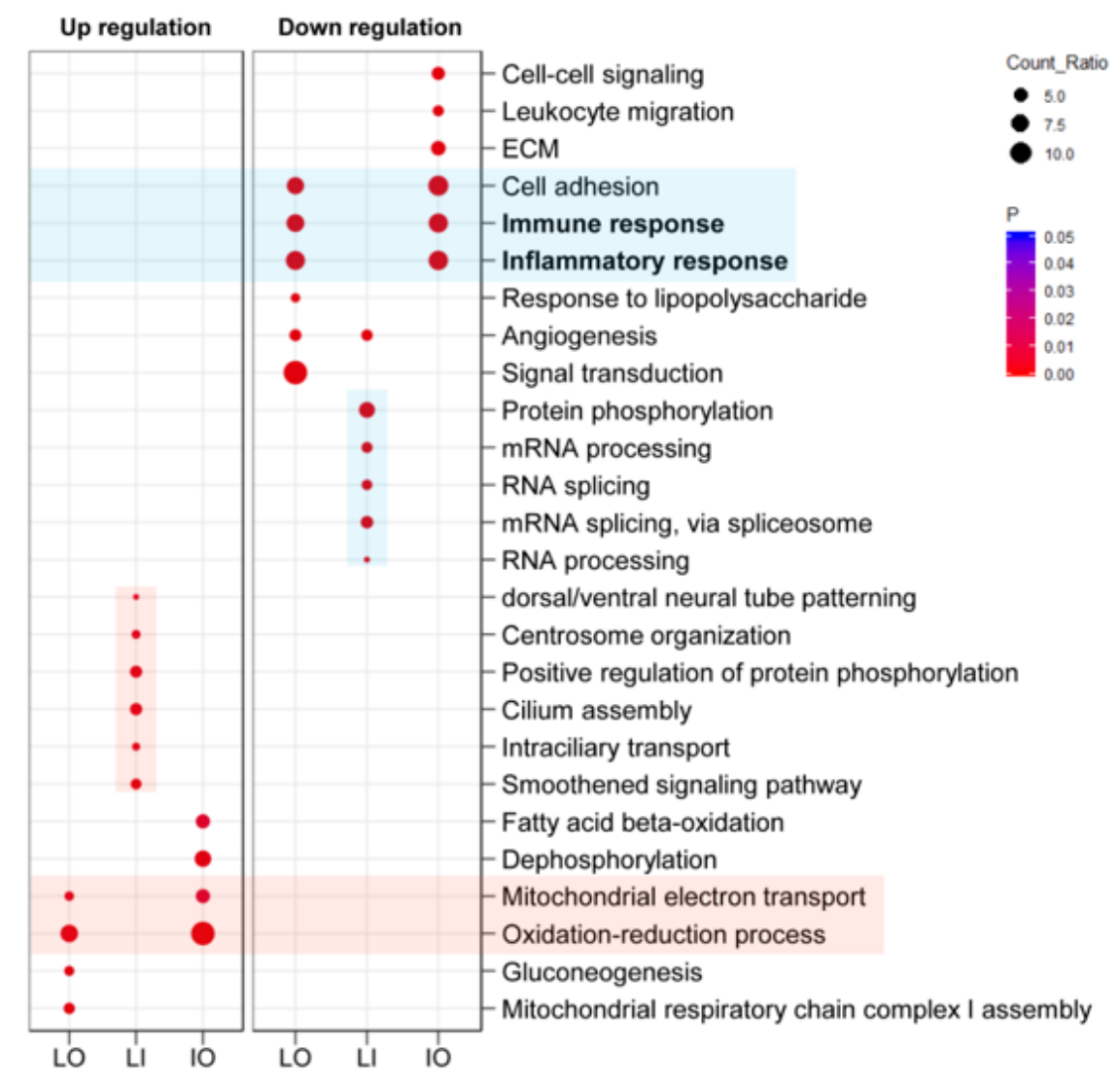

\section{Figure 3}

Three classes of DEGs using the three redefined groups of samples. A. Construction of heatmap coupled with unsupervised hierarchical clustering for three different classes of DEGs. A total of 2,657 'LO-DEGs' (Q $<0.01), 1,474$ 'LI-DEGs' $(P<0.01)$, and 1,324 'IO-DEGs' $(\mathrm{Q}<0.05)$ are used to construct a heatmap coupled with unsupervised clustering (refer to Additional file 4: Table S3). The same notations used for 
Fig. 2A are also used in this heatmap (refer to Fig. 2A legend). B. Analysis of GO functional terms for each of the three classes of DEGs (see Materials and methods). 'LO', 'LI', and 'IO' represent 'LO-DEGs', 'LIDEGs' and 'IO-DEGs', respectively. For each class of DEGs, genes are divided into upregulated genes (i.e., genes with higher levels in obese ACs than in lean ACs) and downregulated genes (i.e., genes with lower levels in obese ACs than in lean ACs). Wide red and blue boxes within the plot indicate upregulated and downregulated genes, respectively, for both 'LO-DEGs' and 'IO-DEGs'. Narrow red and blue boxes within the plot indicate upregulated and downregulated genes, respectively, for the 'LI-DEGs'.

Fig. 4

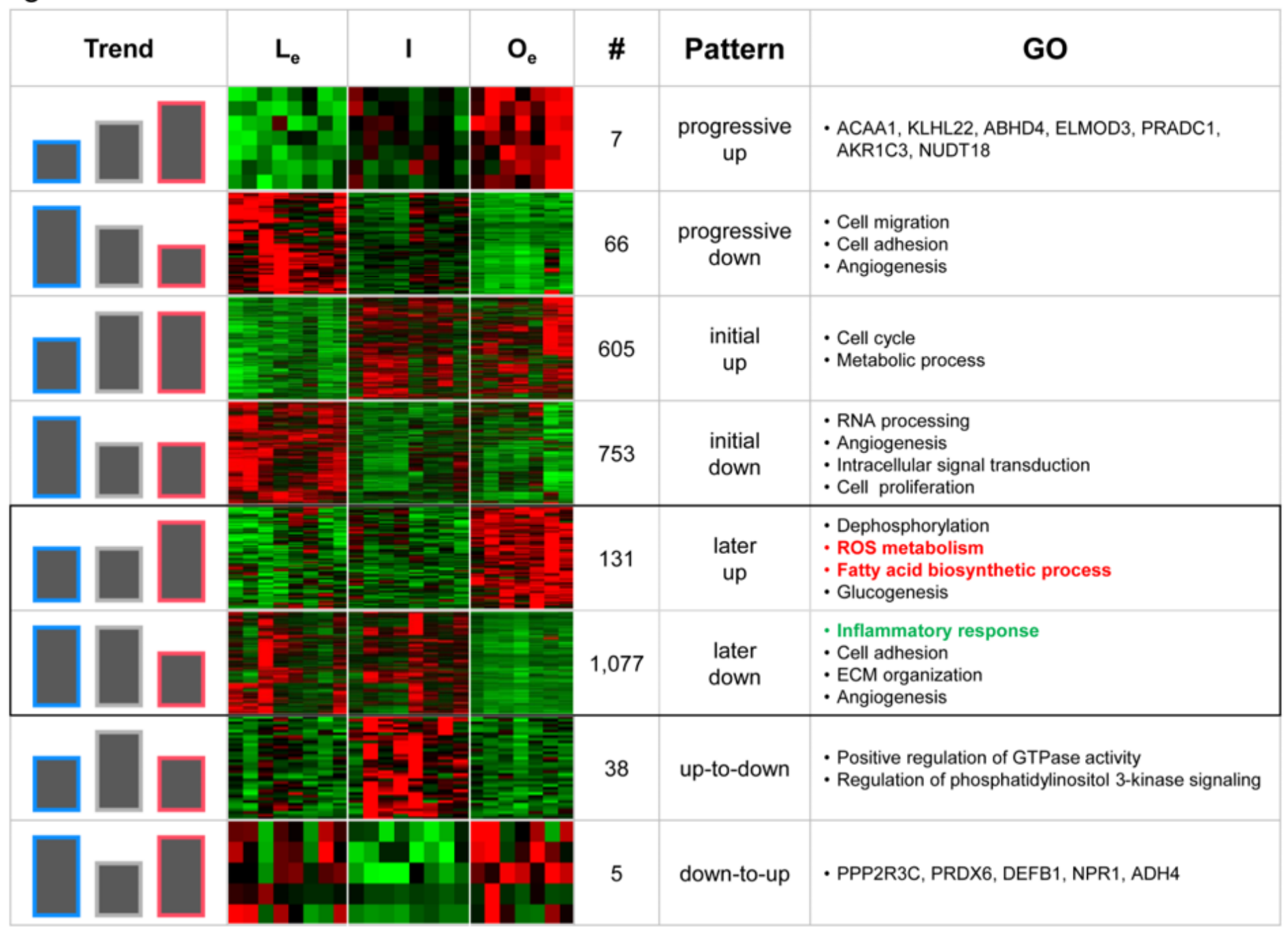

\section{Figure 4}

Examination of changes in gene expression between lean ACs and obese ACs. Changes in gene expression are allocated into eight different categories: 'progressive-up/down', 'initial-up/down' 'laterup/down', and 'up-to-down/down-to-up'. Each category of DEGs is obtained by intersecting 'LI-DEGs' and 'IO-DEGs' (refer to Additional file 6: Figure S3 for the detailed strategy for the subcategorization). A heatmap is generated for each category of DEGs with genes assigned to each category. The bars in the first column represent the trend of gene expression levels in each category, and the colors, blue, gray, and red of the lines surrounding each bar represent genes in the Le, I, and Oe categories, respectively. 
Fig. 5

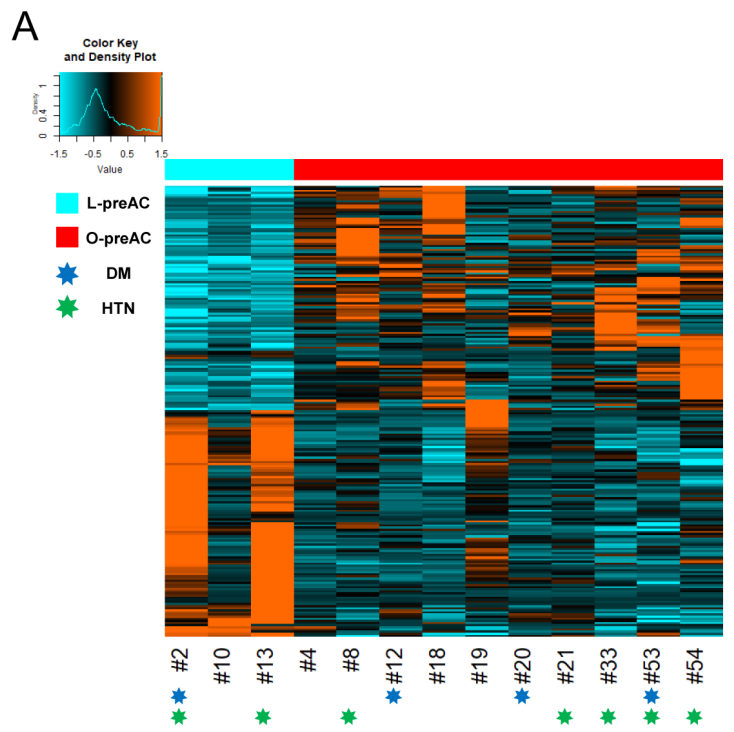

B

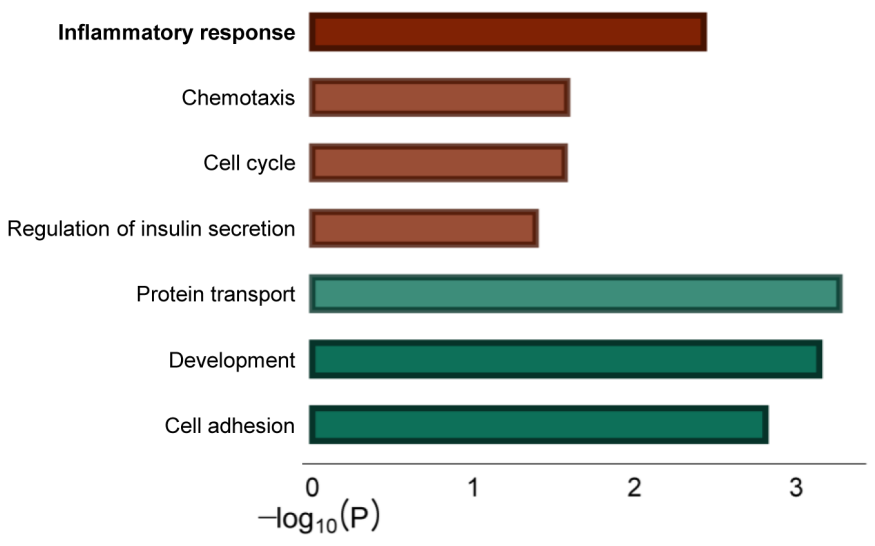

\section{Figure 5}

Differentiation of lean preAC samples and obese preAC samples by 'Class II: preAC-DEGs'. A.

Construction of heatmap accompanied with unsupervised hierarchical clustering. A heatmap coupled with unsupervised hierarchical clustering is generated with a total of 213 'Class II: preAC-DEGs' $(p<0.01)$ (Additional file 2: Table S1). Refer to the notations in the Fig. 2A legend. B. GO analysis of 'preAC-DEGs'; red and green bars represent upregulated (i.e., genes with higher levels in obese preACs than in lean preACs) and downregulated genes (i.e., genes with lower levels in obese preACs than in lean preACs), respectively. Refer to the Fig. $2 \mathrm{~B}$ legend for how to select the functional terms and the meaning of the scale on the bottom. 
Fig. 6

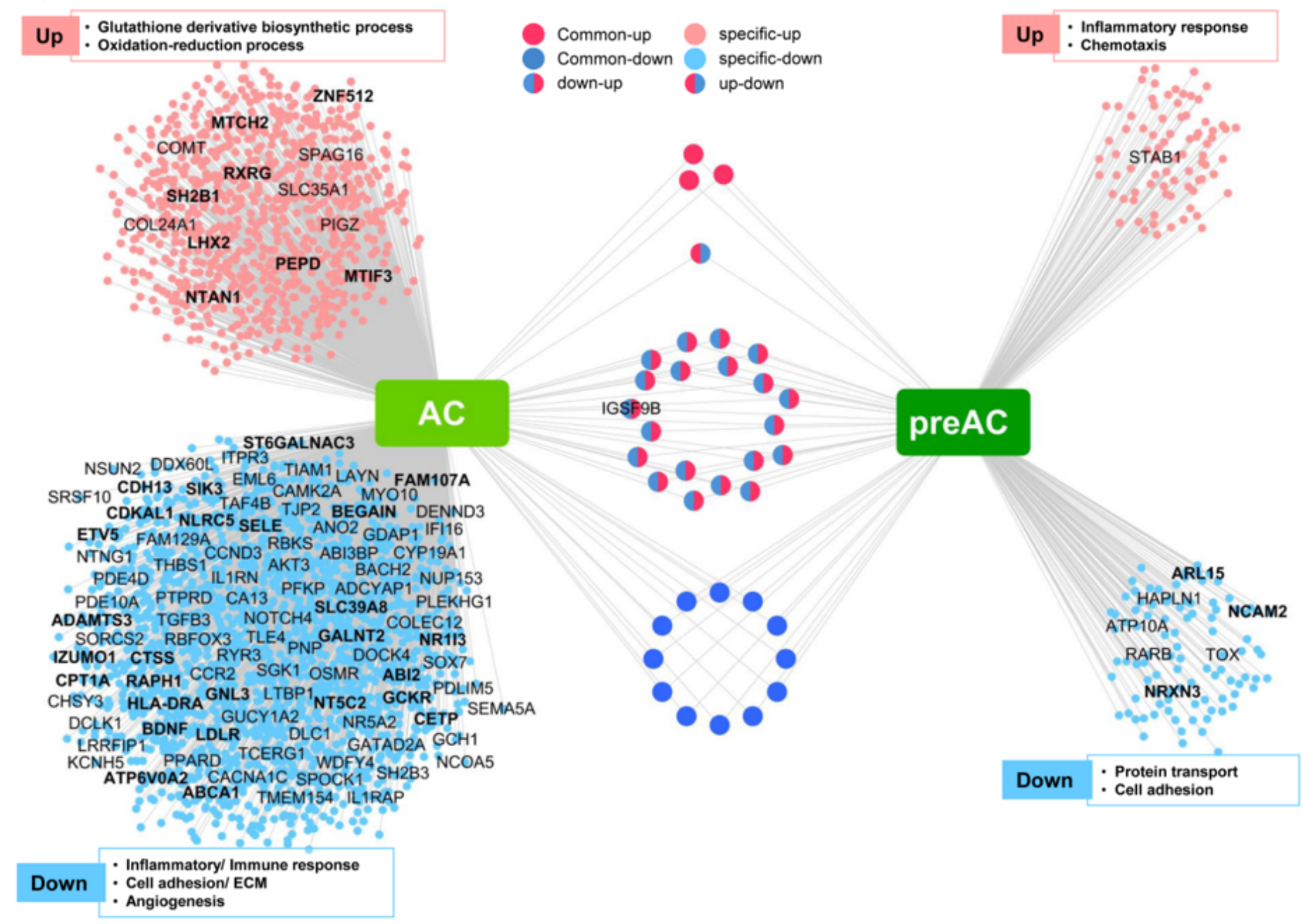

\section{Figure 6}

Integration of DEGs between 'AC-DEGs' and 'preAC-DEGs'. To compare DEGs obtained from ACs with those from preACs, a total of 2,657 'LO-DEGs' and 213 'preAC-DEGs' are intersected; from these, DEGs are divided into five groups depicted in the graph; dots in the blue box represent genes that are expressed at lower levels in obese samples than in lean samples (i.e., downregulated genes), and dots in the pink box represent genes that are expressed at higher levels in obese samples than in lean samples (i.e., upregulated genes). Each dot representing each gene is connected to ACs or preACs accordingly; 'ACspecific-up', 'AC-specific-down', 'preAC-specific-up', 'preAC-specific-down', and 'Common'. The two colored (depicted as blue-red or red-blue) balls in the middle of the graph represent DEGs that are commonly found in both 'LO-DEGs' (i.e., AC-DEGs) and 'preAC-DEGs' but in the opposite directions (i.e., up for 'LODEGs' but down for 'preAC-DEGs' or its inverse). Genes depicted with the gene symbols and gene symbols with the bold face represent the genes that overlap with the genes previously identified as obesityassociated genes by GWAS with $p<5 \times 10-6$ and $p<5 \times 10-8$, respectively. 
Fig. 7

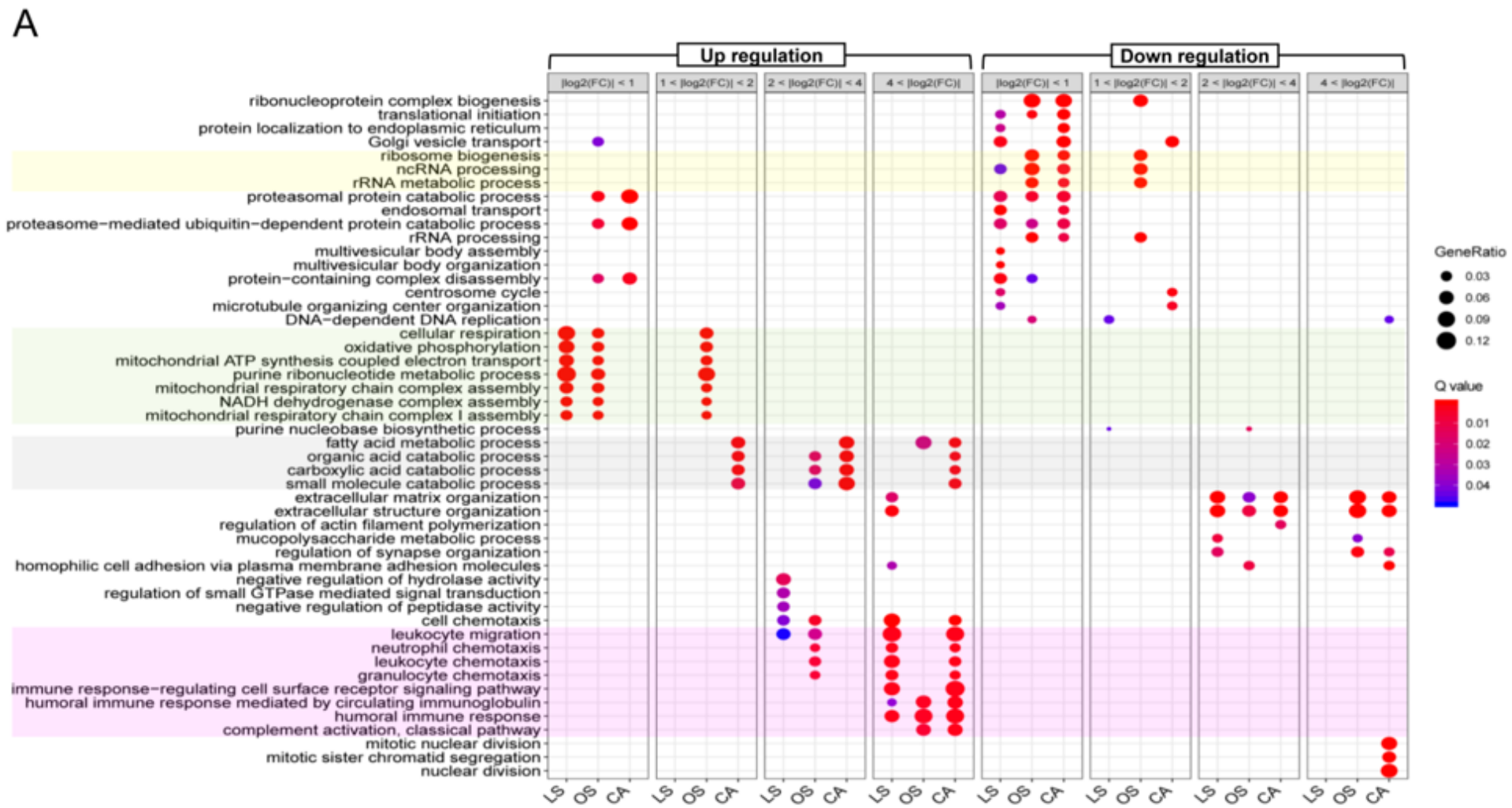

B

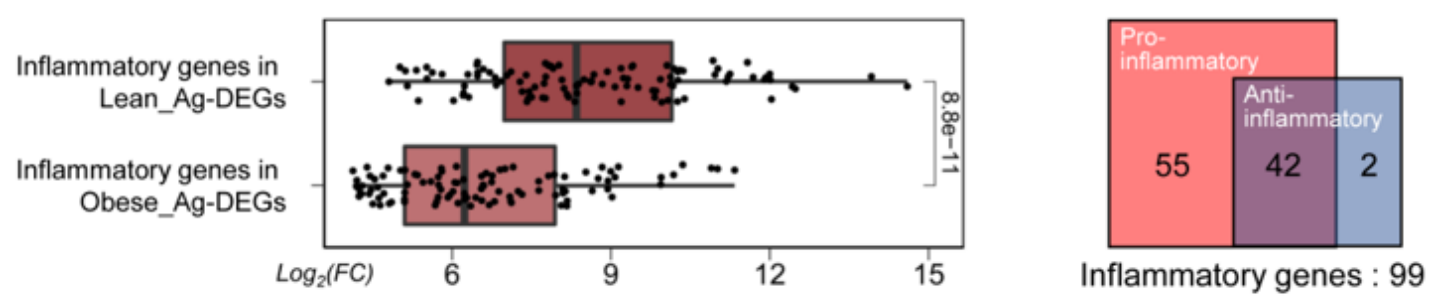

\section{Figure 7}

Analysis of the functions of genes that are significantly altered between lean and obese AC differentiation. A. 'Clusterprofile' analysis of GO functional terms. Lean_Ag-DEGs and Obese_Ag-DEGs are intersected, leading to three subcategories: 'LS', 'OS', and 'CA' (see the main text). For LS, OS, and CA, DEGs are subdivided into upregulated (i.e., genes that are expressed at higher levels in ACs than in preACs) and downregulated genes (i.e., genes that are expressed at lower levels in ACs than in preACs). Upregulation and downregulation are further divided into four groups by considering the log2 fold change in gene expression along with the $Q<0.01$ threshold. Functional enrichment of genes in each class is investigated and plotted by 'Clusterprofiler' (see Materials and methods). Refer to the main text for the meaning of each colored box. B. Left: box plot of expression levels of inflammatory genes in the ' $C A$ ' category. A total of 99 inflammatory genes were found by mapping these genes to annotation on Gene Cards (http://genecards.org). Box plots are constructed using the Log2(FC) values calculated for each of the 99 genes between $\mathrm{AC}$ and preAC samples for lean and obese conditions, respectively. Right: Square Venn diagram showing the numbers of pro-/anti-inflammatory genes. Statistical significance is estimated by Wilcoxon's test. 


\section{Supplementary Files}

This is a list of supplementary files associated with this preprint. Click to download.

- Additionalfile6.pdf

- Additionalfile8.pdf

- Additionalfile5.pdf

- Additionalfile10.pdf

- Additionalfile7.pdf

- Additionalfile11.pdf

- Additionalfile3.pdf

- Additionalfile4.pdf

- Additionalfile9.xlsx

- Additionalfile1.pdf

- Additionalfile2.pdf 